\title{
La situation et les soins des réfugié-e-s mineur-e-s en Suisse
}

\author{
Alain Di Galloa, Hélène Beutler ${ }^{b}$ \\ a $\operatorname{Pr} \operatorname{Dr}$ méd., co-président de la SSPPEA, membre FMH; ${ }^{\mathrm{b}}$ Dre, co-présidente de la SSPPEA, membre FMH
}

Correspondance:

Pr Alain Di Gallo,

co-président de la SSPPEA, Dre Hélène Beutler co-présidente de la SSPEA, SGKJPP/SSPPEA Altenbergstrasse 29 Case postale 686 CH-3000 Berne 8 Tél. 0313138834 Fax 0313138899 sgkjpp[at]psychiatrie.ch
Le nombre des réfugié-e-s mineur-e-s est élevé. En 2015, quelque 40000 demandes d'asile ont été déposées; plus de 10000 concernaient des enfants ou des adolescents. 2700 jeunes réfugié-e-s sont arrivé-e-s en Suisse non accompagné-e-s. En 2016, ils étaient encore 2000. De sexe masculin pour la plupart, nombreux étaient ceux qui étaient exposés à des souffrances psychiques continues et qui souffraient de troubles psychiques. Les manifestations les plus fréquentes sont les symptômes de stress post-traumatique, les dépressions et les troubles anxieux, qui s'expliquent non seulement par les expériences traumatiques vécues dans le pays d'origine mais aussi par les conditions souvent difficiles lors du trajet vers le pays de migration d'accueil.

La Société suisse de psychiatrie et psychothérapie de l'enfant et de l'adolescent (SSPPEA) a rédigé une prise de position dans laquelle elle a formulé les principales exigences en matière de prise en charge psychiatrique de ces enfants et adolescents. La société de discipline médicale vise par ce document à attirer l'attention sur la situation psychique des réfugié-e-s mineur-e-s et à exprimer des exigences relatives au soin de ces jeunes. Une prise en charge psychiatrique précoce et adaptée peut les aider à vivre avec leurs traumatismes psychiques tout en favorisant leur intégration. La prise de position se veut également une recommandation à l'intention des psychiatres suisses de l'enfant et de l'adolescent, ainsi qu'un document de sensibilisation pour les centres d'enregistrement et de procédure et les centres d'accueil pour migrants.

\footnotetext{
Lien vers la prise de position

www.sgkjpp.ch/fr/ $\rightarrow$ Relations publiques $\rightarrow$ Projets \& Thèmes $\rightarrow$ Prise de position
}

Exigences en matière de soins psychiatriques:

- Couvrir les besoins humanitaires essentiels reste primordial: sécurité, dignité humaine, protection contre la discrimination, droit à la formation.

- Les enfants et les adolescent-e-s ont besoin d'un environnement et d'un hébergement favorables à leur développement: pas de séparation des familles, plus grand respect possible de la sphère privée familiale et de l'intimité, garantie des possibilités de jouer et de la scolarisation, possibilité de logement collectif pour les jeunes et séparé des adultes inconnus.

- Dans le cadre de l'examen sanitaire à la frontière, des questions doivent être posées sur les signes de souffrance psychique (maux de tête et de ventre, problèmes d'insomnie, apathie, aboulie, etc.), et au besoin, il convient d'établir un diagnostic. Une évaluation plus approfondie devrait être proposée.

- Dans les centres d'accueil et les lieux d'hébergement pour requérant-e-s d'asile, le personnel doit non seulement être attentif aux signes de maladie somatiques et les connaître, mais disposer aussi des compétences correspondantes pour déceler les signes de maladies psychiques.

- Les médecins en charge de l'examen sanitaire à la frontière et les responsables des centres d'accueil et d'hébergement doivent pouvoir accéder facilement à un point de contact psychiatrique et psychologique.

- Les cliniques/services pédopsychiatriques et les groupes spécialisés de psychiatres et psychologues pour enfants et adolescents au niveau cantonal doivent développer ensemble un dispositif pour les soins de base des réfugié-e-s mineur-e-s et dispenser les soins correspondants.

- Les autorités compétentes doivent mettre à la disposition des personnes intéressées intervenants des services d'interprétariat adaptés. 